\title{
Revista de Neuropsiquiatría de Chile
}

$\mathrm{E}_{\mathrm{s}}^{\mathrm{l}}$ primer número de la Revista de Neuropsiquiatría de Chile, órgano oficial de la Sociedad de Neurología, Psiquiatría y Neurocirugía de Chile, SONEPSYN, vio por primera vez la luz hace 71 años, en 1947. Desde ahí en adelante, la Revista se ha publicado con regularidad de tal modo que hoy es parte de varios sistemas de integración de artículos como son: Scielo, BIREME, EMBASE, SIIC y Latindex. Al igual que SONEPSYN, ha estado orientada a integrar no solo actividades académicas, sino también la investigación y la reflexión de las distintas especialidades médicas relativas al funcionamiento del Sistema Nervioso Central, y en un mundo en el cual las tendencias han sido hacia la especialización y la consecuente segregación, esta Revista y su sociedad científica madre, se han mantenido históricamente como ámbitos en los cuales se ha privilegiado la integración del conocimiento entre las especialidades. De manera muy interesante, en el mundo actual, con la deslumbrante emergencia de las neurociencias, tal integración se ha vuelto naturalmente necesaria, y es así como nuestra Revista de Neuropsiquiatría de Chile se encuentra hoy en situación de responder a tal orientación, principalmente, porque históricamente ésta ha sido su principal norte.

Por cierto, se ha hecho crecientemente necesario integrarse de modo más pleno a la vorágine que supone la difusión de la información y el conocimiento propio del mundo actual, no solo el científico. No es el lugar ni el momento para extenderse acerca de que tal difusión es parte integral del proceso de generación de conocimiento basado en el método científico. Para tal integración son imprescindibles dos condiciones: la primera, publicar en el idioma que hoy se considera universal, el inglés; y segundo, hacerlo por el medio que hoy preferentemente conduce la corriente de informa- ción, el digital. El Directorio de Sonepsyn ha considerado que no puede retrasarse la incorporación de estas condiciones a nuestra augusta Revista, y ha encargado al equipo editorial actual llevar a cabo esa tarea. Nos complace poner en conocimiento no solo de los socios de SONEPSYN sino también de la comunidad científica toda, que el próximo número de la Revista de Neuropsiquiatría de Chile estará alojado, además de su ya clásica versión digital en español en la web de SONEPSYN, en una versión exclusivamente en inglés en el sitio $w w w$. journalofneuropsychiatry.cl. para lo cual, la Revista proveerá de traducción para los artículos recibidos en español.

La difusión del conocimiento en la actualidad ha sufrido un proceso gradual y definido de comercialización. Lamentablemente, ello ha terminado en un cierto grado de erosión de la calidad de la misma difusión. La aparición de revistas con comités editoriales compuestos por especialistas poco calificados, para permitir la publicación de artículos de baja calidad, y el cobro por publicación proporcional al factor de impacto que tiene una revista son ejemplos evidentes y conocidos de lo señalado. La Revista de Neuropsiquiatría de Chile mantendrá especial cuidado en mantener los principios necesarios para no someterse a la distorsión que estamos señalando que, además, son los que han sido recibidos como tradición por parte de SONEPSYN y de los Comités Editoriales que han precedido al actual. Creemos que ésta puede ser una contribución más allá de la estrictamente referida al conocimiento que se publicará.

En definitiva, podemos decir que se trata de una apasionante aventura que emprendemos con el más abierto de los espíritus. Nuestra Revista se incorpora plenamente a la corriente de la información y del conocimiento del mundo actual. 
Esperamos que sean parte de esta aventura todos nuestros asociados y también los autores extranjeros que con frecuencia nos eligen como medio para la difusión de sus trabajos.

Que así sea.
${ }^{1}$ Profesor Asociado de Psiquiatría de la Universidad de Chile.

Jefe de la Unidad de Trastornos Bipolares, Departamento de Psiquiatría Norte Facultad de Medicina Universidad de Chile

Miembro Honorario de la Asociación Mundial

Dr. Luis Risco N. ${ }^{1}$ de Psiquiatría. 